\title{
SEMIGROUPS OF SCALAR TYPE OPERATORS ON BANACH SPACES $\left({ }^{1}\right)$
}

\author{
BY
}

\section{AHMED RAMZY SOUROUR}

\begin{abstract}
The main result is that if $\{T(t): t \geqslant 0\}$ is a strongly continuous semigroup of scalar type operators on a weakly complete Banach space $X$ and if the resolutions of the identity for $T(t)$ are uniformly bounded in norm, then the infinitesimal generator is scalar type. Moreover, there exists a countably additive spectral measure $K(\cdot)$ such that $T(t)=\int \exp (\lambda t) d K(\lambda)$, for $t \geqslant 0$. This is a direct generalization of the well-known theorem of Sz.-Nagy about semigroups of normal operators on a Hilbert space. Similar spectral representations are given for representations of locally compact abelian groups and for semigroups of unbounded operators. Connections with the theory of hermitian and normal operators on Banach spaces are established. It is further shown that $\boldsymbol{R}$ is the infinitesimal generator of a semigroup of hermitian operators on a Banach space if and only if $i R$ is the generator of a group of isometries.
\end{abstract}

1. Introduction. In this paper we study strongly continuous semigroups $\{T(t): t \geqslant 0\}$ of scalar type operators on a Banach space and extend some wellknown results about semigroups of normal or selfadjoint operators on a Hilbert space. The main result (Theorem 5.3) generalizes Sz.-Nagy's theorem about semigroups of normal operator [12, Theorem 22.4.2] or [19, $\S \mathrm{XI} 3]$. We do not assume that the resolutions of the identity for the operators $T(t)$ generate a bounded Boolean algebra of projections; this is obtained as a result. If one assumes this, a much simpler proof of the main theorem could be given. However, this would be quite restrictive since in general the resolutions of the identity for two commuting scalar type operators do not always generate a bounded Boolean algebra of projections, even on a reflexive space (see [16]).

In $\S 6$ we extend a theorem of Foias [11] on semigroups of scalar type operators on a Hilbert space. In $\S 7$ we study the continuity of $f(T(t))$, as a

Presented to the Society, January 25, 1973; received by the editors June 21, 1973. AMS (MOS) subject classifications (1970). Primary 47D05, 47D10, 47B40.

Key words and phrases. Semigroup of operators, spectral operator, scalar type operator, infinitesimal generator.

(1) This paper is taken from the author's $\mathrm{Ph}$. D. thesis written at the University of Illinois under the direction of Earl Berkson, whose valuable guidance is appreciated by the author. Research supported by NSF GP 28577. 
function of $t$, for a wide class of functions $f$. This gives a result on the continuity of the resolutions of the identity for $T(t)$ as a function of $t$. In $\S 8$ it is proved that a strongly continuous semigroup of hermitian operators on a Banach space has a self-conjugate infinitesimal generator $\boldsymbol{A}$ (i.e., $i \boldsymbol{A}$ generates a group of isometries). This extends the theorem of Hille and Sz.-Nagy on semigroups of selfadjoint operators on a Hilbert space [12, Theorem 22.3.1] or [19, §XI 2]. In $\S 9$ we give a spectral representation for semigroups of unbounded scalar type operators with real spectra. In $\S 10$ the Godement-Naimark theorem about unitary representations of locally compact abelian groups is extended to the case of representations by circled scalar type operators. It is further shown $(\S 11)$ that the functional calculus of Hille-Phillips-Balakrishnan and the fractional powers of Yosida, defined for generators of uniformly bounded semigroups, agree with the most natural definitions when the generator is scalar type.

In what follows $X$ will be a complex Banach space with dual space $X^{*}$. An operator in $X$ will be a linear transformation (not necessarily bounded) with domain and range contained in $X . B(X)$ is the algebra of all bounded operators on $X$. We shall denote the domain, spectrum, resolvent set, and resolvent (evaluated at $\lambda$ ) by $D(T), \sigma(T), \rho(T)$, and $R(\lambda ; T)$ respectively. If $T$ and $S$ are operators in $X$, we say that $T$ is an extension of $S$ (written $T \supseteq S$ or $S \subseteq$ $T)$ if $D(T) \supseteq O(S)$ and $T x=S x$ for every $x \in O(S)$.

Our terminology concerning semigroups of operators will be that of $[8$, Chapter VIII]. For definitions and results on spectral operators, we refer to $[8$, Part III] .

(1.1) Definition. If $T \in B(X)$, the numerical range $V(T)$ of $T$ is defined by

$$
V(T)=\left\{x^{*} T x: x \in X, x^{*} \in X^{*},\left\|x^{*}\right\|=\|x\|=x^{*}(x)=1\right\} .
$$

This is what is called "the spatial numerical range" in [6], and differs from, though is closely related to, the numerical range defined by Lumer [14] via semiinner-products.

(1.2) Definition. If $T \in B(X)$, then $T$ is said to be hermitian if the numerical range of $T$ is real.

This notion was shown to coincide with the one previously introduced by Vidav in [20], namely, that

$$
\|I+i t T\|=1+o(t) \quad \text { as } t \rightarrow 0, \quad t \text { real. }
$$

It is obvious that, on a Hilbert space, the hermitian operators are the selfadjoint operators.

A family $F$ of bounded operators on $X$ is said to be hermitian-equivalent if there is an equivalent renorming of $X$ under which all the operators in $F$ become hermitian. 
(1.3) Definition. Let $A$ be a closed subalgebra of $B(X)$ containing the identity $I$. Then $A$ is said to be a $V^{*}$-algebra if every operator $T \in A$ can be written as $R+i J$ with $R$ and $J$ hermitian operators in A. A will be understood to be equipped with the (Vidav-)involution ${ }^{*}: R+i J \rightarrow R-i J$.

A theorem of Vidav [20] has been sharpened by Berkson, Glickfeld and Palmer (see [6]) to the following form.

(1.4) THEOREM. A is a $V^{*}$-algebra if and only if A is a $C^{*}$-algebra under the Vidav-involution.

2. Semigroups with scalar type generator. We prove the equivalence of the condition that the infinitesimal generator be of scalar type with the existence of a certain representation for all the operators in the semigroup as integrals with respect to one spectral measure.

(2.1) THEOREM. Let $\{T(t)\}$ be a strongly continuous semigroup of operators on $X$. Then there is a countably additive spectral measure $K(\cdot)$ on the Borel sets of the plane with

$$
T(t)=\int e^{\lambda t} d K(\lambda), \quad t \geqslant 0,
$$

if and only if the infinitesimal generator $C$ of $\{T(t)\}$ is a scalar type operator. If this is the case, $K(\cdot)$ is uniquely determined as the resolution of the identity for $C$, and each $T(t)$ is a scalar type operator.

Proof. The "if" part is Theorem 3.1 of [4]. To prove the converse, assume $T(t)=\int e^{\lambda t} d K(\lambda), t \geqslant 0$. Since $T(1)$ is a bounded operator, the exponential function must be $K(\cdot)$-essentially bounded. Hence there is a real number $\gamma$ such that $K(\{\lambda: \operatorname{Re} \lambda>\gamma\})=0$. Let $A$ be defined by

$$
\begin{aligned}
O(A) & =\left\{x: \lim _{n \rightarrow \infty} \int_{e_{n}} \lambda d K(\lambda) x \text { exists }\right\}, \\
A x & =\lim _{n \rightarrow \infty} \int_{e_{n}} \lambda d K(\lambda) x, \quad x \in D(A),
\end{aligned}
$$

where $e_{n}=\{\lambda:|\lambda| \leqslant n\}$. Then $A$ is a scalar type operator with resolution of the identity $K(\cdot)$, and $\sigma(A) \subseteq\{\lambda: \operatorname{Re} \lambda \leqslant \gamma\}$. If $\mu>\gamma$, then $R(\mu ; A)=$ $\int(\mu-\lambda)^{-1} d K(\lambda)$ by [8, XVIII, $\left.2.11(\mathrm{~h})\right]$.

On the other hand, if $\mu$ is sufficiently large, then

$$
R(\mu ; C) x=\int_{0}^{\infty} e^{-\mu t} T(t) x d t, \quad x \in X,
$$

by $[8$, VIII 1.11$]$. Therefore

$$
x^{*} R(\mu ; C) x=\int_{0}^{\infty} \int_{\operatorname{Re} \lambda<\gamma} e^{(\lambda-\mu) t} d\left(x^{*} K(\lambda) x\right) d t, \quad x \in X, x^{*} \in X^{*} .
$$


An application of Fubini's theorem gives

$$
x^{*} R(\mu ; C) x=x^{*} \int \frac{1}{\mu-\lambda} d K(\lambda) x,
$$

and hence $R(\mu ; C)=R(\mu ; A)$, therefore $C=A$. This proves the "only if" part as well as the last sentence of the theorem.

(2.2) COROLlARY. Let $\{T(t)\}$ be a strongly continuous semigroup of operators on $X$ with scalar type infinitesimal generator $C$ whose resolution of the identity is $K(\cdot)$. Then $S \in B(X)$ commutes with every $T(t)$, for $t \geqslant 0$, if and only if $S$ commutes with every projection $K(\delta)$.

Proof. The "if" part follows easily from Theorem 2.1. Assume $S \in B(X)$ commutes with every $T(t)$. Let $\mu$ be sufficiently large so that $R(\mu ; C)=$ $\int_{0}^{\infty} e^{-\mu t} T(t) d t$. Therefore $S$ commutes with $R(\mu ; C)$, and hence with all the projections in the range of its resolution of the identity. But $R(\mu ; C)=$ $\int(\mu-\lambda)^{-1} d K(\lambda)$; hence $S$ commutes with all projections of the form $K(\{\lambda: 1 /(\mu-\lambda) \in \delta\}$, i.e., with every $K(\sigma)$ with $\mu \notin \sigma$. But $K(\{\mu\})=0$ since $\mu \in \rho(C)$; hence $S$ commutes with every $K(\sigma)$. This completes the proof.

3. Semigroups of positive scalar type operators. A real (respectively positive) operator is an operator whose spectrum is real (respectively nonnegative). If all the operators in a semigroup are real operators, then they are automatically positive since $\sigma(T(2 t))=[\sigma(T(t))]^{2}$ for every $t \geqslant 0$. We give a generalization of the Hille-Sz.-Nagy theorem. This is proved for all Banach spaces (weakly complete or not), and without any assumptions about the uniform boundedness of the resolutions of the identity for the operators in the semigroup.

(3.1) THEOREM. Let $\{R(t)\}$ be a strongly continuous semigroup of positive scalar type operators on $X$. Then the infinitesimal generator $A$ is scalar type with spectrum contained in some interval $\left(-\infty, \omega_{0}\right]$. Moreover there is a countably additive spectral measure $G(\cdot)$ defined on the Borel sets of the real line such that

$$
R(t)=\int_{-\infty}^{\omega_{0}} e^{\lambda t} d G(\lambda), \quad t \geqslant 0
$$

$G(\cdot)$ is uniquely determined as the resolution of the identity for $A$.

Proof. Let $E_{t}(\cdot)$ be the resolution of the identity for $R(t)$. Let $n$ be any positive integer, then

$$
R(1)=R(1 / n)^{n}=\int_{0}^{\infty} \lambda^{n} d E_{1 / n}(\lambda)
$$


Hence $E_{1}(\delta)=E_{1 / n}\left(\left\{\lambda: \lambda^{n} \in \delta\right\}\right)$ for any Borel set $\delta$ of the real line, therefore $E_{1 / n}(\delta)=E_{1}\left(\delta^{n}\right)$, and $R(1 / n)=\int \lambda^{1 / n} d E_{1}(\lambda)$.

Let $m$ be an arbitrary positive integer, then

$$
R(m / n)=R(1 / n)^{m}=\int \lambda^{m / n} d E_{1}(\lambda) .
$$

By the strong continuity of $\{R(t)\}$, we get

$$
R(t)=\int \lambda^{t} d E_{1}(\lambda), \quad t>0 .
$$

$E_{1}(\{0\})=0$, for if $x \in E_{1}(\{0\}) X$, then $R(t) x=\int \lambda^{t} d E_{1}(\lambda) E_{1}(\{0\}) x=0$ for any $t>0$, and hence by the strong continuity $x=0$.

Define $G(\delta)=E_{1}(\exp \delta)$ for any Borel set $\delta$. Thus $G(\cdot)$ is a countably additive spectral measure since $E_{1}(\{0\})=0$. Moreover

$$
R(t)=\int_{-\infty}^{\omega_{0}} e^{\lambda t} d G(\lambda), \quad t>0,
$$

where $\omega_{0}=\log \max \sigma(R(1))$. The same is obviously true for $t=0$. It follows from Theorem 2.1 that $A$ is a scalar type operator, and that $G(\cdot)$ is uniquely determined as the resolution of the identity for $A$.

(3.2) CoRollary. With the same notation as in Theorem 3.1, let $S \in$ $B(X)$, then the following conditions are equivalent.

1. $S$ commutes with $R\left(t_{0}\right)$ for some $t_{0}>0$.

2. $S$ commutes with every $R(t)$ for $t \geqslant 0$.

3. $S$ commutes with every projection $G(\delta)$.

Proof. It follows from the proof of Theorem 3.1 that the range of $G(\cdot)$ is the same as the range of $E_{t_{0}}(\cdot)$, for any $t_{0}>0$; hence (1) implies (3). Other implications are obvious.

(3.3) COROLlaRY. Let $\{R(t)\}$ be a strongly continuous semigroup of positive scalar type operators on $X$. Then there is an equivalent renorming of $X$ under which all the operators $R(t), t \geqslant 0$, as well as all the projections in their resolutions of the identity become hermitian. If $X$ is given as a Hilbert space, the new norm can be chosen to be a Hilbert space norm, and hence the operators $R(t), t \geqslant 0$, become selfadjoint.

Proof. Let $G(\cdot)$ be as in Theorem 3.1. Then, by a result of Berkson [3, Lemma 2.3], there is an equivalent renorming of $X$ under which all the projections $G(\delta)$ become hermitian. Hence all the operators $R(t), E_{t}(\delta)$ become hermitian. The last sentence in the corollary follows from [15, Theorem 6]. 
4. Polar decomposition. In the case of a Hilbert space, a semigroup $\{N(t)\}$ of normal operators can be written as the product of a semigroup of positive selfadjoint operators and a semigroup of unitary operators. We prove here an analogous result for semigroups of scalar type operators on a weakly complete space $X$. First we prove a lemma establishing a "polar decomposition" for a single bounded scalar type operator on a Banach space $X$. This decomposition was proved by Foias [11] for scalar type operators on a Hilbert space by a proof valid only in a Hilbert space. A bounded operator is said to be circled if its spectrum is included in the unit circle $\{\lambda:|\lambda|=1\}$.

(4.1) Lemma. Let $T$ be a bounded scalar type operator on a Banach space $X$ with resolution of the identity $E(\cdot)$. Then there are unique operators $R, U$ such that

(i) $T=R U=U R$;

(ii) $U$ is a circled scalar type operator;

(iii) $R$ is a positive scalar type operator;

(iv) $U E(0)=E(0) U=E(0)$.

The decomposition expressed by the double equality (i) is unique under (ii)', (iii), and (iv), where (ii)' is the condition that $U$ is only circled (not assumed to be scalar type). Moreover $R$ is uniquely determined by (i), (ii)', and (iii) only.

Proof. Let

$$
R=\int|\lambda| d E(\lambda), \quad U=\int u(\lambda) d E(\lambda)
$$

where $u(\lambda)=\lambda /|\lambda|$ for $\lambda \neq 0$, and $u(0)=1$. Properties (i)-(iv) are then easily verified.

To prove the uniqueness, let $R_{1}$ and $U_{1}$ be a pair of operators satisfying (i), (ii)', and (iii). Since $R_{1}$ and $U_{1}$ commute with $T$, they commute with $E(\delta)$ for every Borel set $\delta$, and hence with $R$ and $U$. Let $A$ be the full commutative algebra generated by $R, U, R_{1}$ and $U_{1}$ and let ${ }^{\wedge}$ be the Gelfand mapping of $A$. Then $\hat{T}=\hat{R} \hat{U}=\hat{R}_{1} \hat{U}_{1}$. The spectrum of any operator in $A$ (viewed as a member of $A$ ) is the same as its spectrum if considered as a member of $B(X)$, since $A$ is a full subalgebra. Thus $\hat{R}$ and $\hat{R}_{1}$ are positive-valued functions, while $\hat{U}$ and $\hat{U}_{1}$ take values in the unit circle. Therefore $\hat{R}=$ $|\hat{T}|=\hat{R}_{1}$ and hence $R-R_{1}$ is quasi-nilpotent, but the commuting operators $R$ and $R_{1}$ are each scalar type. It follows from the uniqueness of the canonical decomposition of spectral operators [8, Theorem XV 4.5] that $R_{1}=R$.

If condition (iv) is also satisfied, then for any $x \in X$,

$$
T\left(U-U_{1}\right) x=U R\left(U-U_{1}\right) x=U(T-T) x=0 .
$$


Thus $\left(U-U_{1}\right) x$ belongs to the null space of $T$, which is the range of $E(0)$. Thus

$$
\left(U-U_{1}\right) x=E(0)\left(U-U_{1}\right) x=0,
$$

by condition (iv). Therefore, $U=U_{1}$ and the proof of the lemma is complete.

REMARK. In [10, Theorem 5.2], Foguel obtained the above decomposition, but claimed the uniqueness without condition (iv). The following counterexample shows that this is false even if $T$ is a normal operator on a Hilbert space, $R$ positive selfadjoint and $U$ unitary.

(4.2) Counterexample. Let $X$ be $l^{2}$ and let

$$
\begin{gathered}
T\left(x_{1}, x_{2}, x_{3}, \cdots\right)=\left(0, x_{2}, x_{3}, \cdots\right), \\
R=T, \quad U_{1}=I, \\
U_{2}\left(x_{1}, x_{2}, x_{3}, \cdots\right)=\left(\alpha x_{1}, x_{2}, x_{3}, \cdots\right),
\end{gathered}
$$

where $\alpha$ is any complex number with $|\alpha|=1$. Then $T=R U_{i}=U_{i} R, i=$ 1,$2 ; T=R$ is positive; $U_{1}$ and $U_{2}$ are unitary.

Note that condition (iv) is automatically satisfied when $T$ is one-to-one, since $E(\{0\})=0$ in this case.

(4.3) Definition. Let $T$ be a scalar type operator, $R$ and $U$ the unique operators defined in Lemma 4.1. Then $R$ will be called the positive part of $T$, $U$ the circled part of $T$ and the decomposition $T=R U$ will be called the polar decomposition of $T$.

(4.4) THEOREM. Let $\{T(t)\}$ be a strongly continuous semigroup of scalar type operators on $X$ having their resolutions of the identity uniformly bounded in norm, and let $R(t)$ and $U(t)$ be the positive part and the circled part of $T(t)$ respectively. Then

1. $\{T(t)\} \cup\{R(t)\} \cup\{U(t)\}$ is a commutative family;

2. each of $\{R(t)\}$ and $\{U(t)\}$ is a strongly continuous semigroup;

3. there exists a countably additive spectral measure $G(\cdot)$ on the Borel sets of the real line such that $R(t)=\int e^{\lambda t} d G(\lambda), t>0$.

Moreover if $\{T(t)\}$ is of type $\omega_{0}$, then $\{R(t)\}$ is of the same type $\omega_{0}$, and $G\left(\left(-\infty, \omega_{0}\right]\right)=I$.

Proof. Let $E_{t}(\cdot)$ be the resolution of the identity for $T(t)$ and let $\left\|E_{t}(\delta)\right\| \leqslant M$ for all $t \geqslant 0$ and all Borel sets $\delta$.

First we prove that $T(t)$ is one-to-one for every $t \geqslant 0$ and hence condition (iv) of Lemma 4.1 is automatically satisfied for the operators $T(t)$. We will prove this for $T(1)$, a similar proof holding for any $t$. If $n$ is any integer $>1$, then 


$$
T(1)=T(1 / n)^{n}=\int \lambda^{n} d E_{1 / n}(\lambda),
$$

and hence $E_{1}(\delta)=E_{1 / n}\left(\left\{\lambda_{:} \lambda^{n} \in \delta\right\}\right)$, for every Borel set $\delta$. In particular $E_{1}(0)=E_{1 / n}(0)$. If $x \in E_{1}(0) X$, then $T(1 / n) x=0$ for all $n>1$, and hence by strong continuity $x=T(0) x=0$, i.e., $E_{1}(\{0\})=0$.

Let $F_{t}(\cdot)$ be the resolution of the identity of $R(t)$ and let

$$
S(t)=\int_{\{\lambda: \lambda>0\}} \lambda^{t} d F_{1}(\lambda) .
$$

We begin by showing that $S(t)=R(t)$ for $t$ rational. Note that for any positive integer $n$, and any $t \geqslant 0, T(n t)=(T(t))^{n}=(R(t))^{n}(U(t))^{n}$ and an application of Lemma 4.1 gives $R(n t)=(R(t))^{n}, U(n t)=(U(t))^{n}$. Therefore

$$
R(1)=R(1 / n)^{n}=\int \lambda^{n} d F_{/ n}(\lambda),
$$

and hence $F_{1}(\delta)=F_{1 / n}\left\{\lambda: \lambda^{n} \in \delta\right\}$, for every Borel set $\delta$. Therefore if $\delta$ is any Borel subset of the set of nonnegative real numbers $F_{1 / n}(\delta)=F_{1}(\{\lambda$ :

$\left.\left.\lambda^{1 / n} \in \delta\right\}\right)$. But the same is true for the resolution of the identity of $S(1 / n)$ and hence $S(1 / n)=R(1 / n)$. It follows that $S(m / n)=R(m / n)$ for any positive integers $m$ and $n$.

Now we prove the existence of circled operators $V(t)$ such that $T(t)=$ $S(t) V(t)=V(t) S(t)$, and that $\{V(t): t \geqslant 0\}$ is a strongly continuous semigroup. The uniqueness of the polar decomposition would then imply that $R(t)=S(t)$, $V(t)=U(t)$. Let

$$
e_{n}=[1 / n, \infty), \quad X_{n}=F_{1}\left(e_{n}\right) X, \quad X_{0}=\bigcup_{n=1}^{\infty} F_{1}\left(e_{n}\right) X .
$$

Then $X_{0}$ is dense in $X$ since $F_{1}\left(e_{n}\right) \rightarrow I$ strongly. Also for any $t$ and any positive integer $n, S(t) \mid X_{n}$ is invertible, hence the range of $S(t)$ contains $X_{0}$. Thus $S(t)$ is one-to-one with dense range, and thus $V(t)=T(t) S(t)^{-1}$ exists as a linear operator defined on $S(t) X$, not as yet known to be bounded. We will show that $V(t)$ extends to a bounded operator (necessarily unique), also denoted by $V(t)$. This is trivial for $t$ rational since then

$$
V(t) x=T(t) S(t)^{-1} x=T(t) R(t)^{-1} x=U(t) x, \quad x \in S(t) X .
$$

If $t$ is irrational, let $\left\{t_{n}\right\}$ be a sequence of rational numbers with $t_{n} \rightarrow t$. Then $\left(S\left(t_{n}\right)\right)^{-1} x \rightarrow(S(t))^{-1} x$ for $x \in X_{0}$ since for $x \in X_{k}$,

$$
\left(S\left(t_{n}\right)\right)^{-1} x=\int_{1 / k}^{\infty} \lambda^{-t_{n}} d F_{1}(\lambda) x, \quad(S(t))^{-1} x=\int_{1 / k}^{\infty} \lambda^{-t} d F_{1}(\lambda) x .
$$

Therefore $T\left(t_{n}\right)\left(S\left(t_{n}\right)\right)^{-1} x \rightarrow T(t)(S(t))^{-1} x$ since $\left\{\left\|T\left(t_{n}\right)\right\|\right\}$ is bounded by the Banach-Steinhaus theorem, and $T\left(t_{n}\right) \rightarrow T(t)$ strongly. Hence $U\left(t_{n}\right) x \rightarrow$ $V(t) x, x \in X_{0}$. But, for any $x \in X$, 


$$
\left\|U\left(t_{n}\right) x\right\|=\left\|\int u(\lambda) d E_{t_{n}}(\lambda) x\right\| \leqslant 4 M\|x\| .
$$

Thus, by [8, II 1.18], $U\left(t_{n}\right)$ converges strongly to a bounded operator on $X$, still called $V(t)$ by abuse of language; moreover $\|V(t)\| \leqslant 4 M$.

Also $V(t)$ commutes with $E_{s}(\delta)$ for any $s>0$ and any Borel set $\delta$, since $U\left(t_{n}\right)$ does. It follows that $V(t)$ commutes with $T(s)$ and $S(s)$, in particular with $S(t)$. Hence

$$
T(t)=S(t) V(t)=V(t) S(t) .
$$

$\{V(t)\}$ is a semigroup, for if $x \in X_{0}, t, s$ any positive real numbers, then

$$
\begin{aligned}
V(t+s) x & =T(t+s) S(t+s)^{-1} x=T(t) T(s) S(t)^{-1} S(s)^{-1} x \\
& =T(s) V(t) S(s)^{-1} x=V(t) V(s) x .
\end{aligned}
$$

Next we prove that the $V(t)$ are circled. This is obvious for rational $t$. If $t$ is irrational, let $t_{n}$ be a sequence of rational numbers with $t_{n} \rightarrow t$. Since the operators $U\left(t_{n}\right)$ are circled, and $U\left(t_{n}\right) \rightarrow V(t)$ strongly, it is enough to show that $\rho(V(t))$ intersects each component of the complement of the unit circle (by the argument in [4, Theorem 2.2]). Therefore it is enough to show that $0 \in \rho(V(t))$. Let $s>0$ be such that $t+s$ is rational. Then $V(t) V(s)=$ $V(t+s)=U(t+s)$ is invertible and hence $V(t)$ is invertible since $V(t)$ and $V(s)$ commute.

Since $T(t)=S(t) V(t)$, and $S(t), V(t)$ commute, the uniqueness of the polar decomposition shows that for all $t>0$

$$
R(t)=S(t)=\int \lambda^{t} d F_{1}(\lambda), \quad U(t)=V(t) .
$$

We proved above that $U\left(t_{n}\right) \rightarrow V(t)$ strongly if $\left\{t_{n}\right\}$ is a sequence of rational numbers, $t_{n} \rightarrow t$. The same proof now gives $U\left(t_{n}\right) \rightarrow U(t)$ strongly for any sequence $\left\{t_{n}\right\}$ with $t_{n} \rightarrow t$, and hence $\{U(t)\}$ is strongly continuous. The weak (and hence strong) continuity of the semigroup $\{R(t)\}$ is evident.

Part (3) follows from Theorem 3.1. We have

$$
\|T(t)\| \leqslant\|R(t)\|\|U(t)\| \leqslant 4 M\|R(t)\|,
$$

and

$$
\|R(t)\| \leqslant\left\|U(t)^{-1}\right\|\|T(t)\| \leqslant 4 M\|T(t)\| .
$$

It follows that $\{R(t)\}$ and $\{T(t)\}$ are of the same type $\omega_{0}$. This ends the proof of the theorem.

5. The main result. The special case where the operators $T(t)$ are circled was considered by Berkson [4]. His result generalizes the well-known theorem of M. H. Stone on unitary groups of operators on a Hilbert space (see [12, p.598] or [19]). We will use Berkson's result and hence state it here for convenience. 
(5.1) THEOREM [4]. If $\{U(t):-\infty<t<\infty\}$ is a strongly continuous group of circled scalar type operators on a weakly complete Banach space $X$ such that their resolutions of the identity are uniformly bounded in norm, then the infinitesimal generator $B$ is a scalar type operator, and $U(t)=\int_{-\infty}^{\infty} e^{i t \lambda} d H(\lambda)$, where $H(\cdot)$ is the resolution of the identity for $-i B$.

We note that the corresponding result for semigroups (rather than groups) of circled operators is a direct consequence of the above since every such semigroup $\{U(t): t \geqslant 0\}$ can be extended to a strongly continuous group satisfying all the conditions above by defining $U(t)=U(-t)^{-1}$ for $t<0$.

The following lemma is needed for the next result.

(5.2) LEMma. Let $\{T(t)\}$ be a uniformly continuous semigroup of scalar type operators on $X$. Then the infinitesimal generator $C$ is a scalar type operator. Let $K(\cdot)$ be the resolution of the identity for $C$ and $E_{t}(\cdot)$ the resolution of the identity for $T(t)$. Then the range of $K(\cdot)$ is the same as the range of $E_{t}(\cdot)$ for $t>0$ small enough, and hence

$$
\sup \{\|K(\delta)\|: \delta \in \Sigma\}=\sup \left\{\left\|E_{t}(\delta)\right\|: \delta \in \Sigma\right\},
$$

for such $t$, where $\Sigma$ is the class of Borel sets of the complex plane.

Proof. We can take $t(>0)$ small enough so that $\|T(t)-I\|<1$, and hence $\log T(t)=-\Sigma_{n=1}^{\infty}(I-T(t))^{n} / n$ is a (bounded) operator. It is well known (see, e.g., [8, proof of VIII 1.2]) that the infinitesimal generator $C$ is given by $C=t^{-1} \log T(t)$, and hence $C=\int t^{-1} \log \lambda d E_{t}(\lambda)$. Therefore $C$ is a scalar type operator with resolution of the identity $K(\cdot)$ given by

$$
K(\delta)=E_{t}\left\{\lambda: t^{-1} \log \lambda \in \delta\right\}, \quad \delta \in \Sigma .
$$

Moreover, $T(t)=\exp t C$, and hence

$$
E_{t}(\alpha)=K\left\{\lambda: e^{t \lambda} \in \alpha\right\}, \quad \alpha \in \Sigma .
$$

This proves the lemma.

Next we take up the main theorem.

(5.3) THEOREM. Let $\{T(t): t \geqslant 0\}$ be a strongly continuous semigroup of scalar type operators on a weakly complete Banach space $X$, with the operators $T(t), t \geqslant 0$, having their resolutions of the identity uniformly bounded in norm. Then the infinitesimal generator $C$ is scalar type. There exists a spectral measure $K(\cdot)$ on the Borel sets of the plane such that

$$
T(t)=\int e^{t z} d K(z), \quad t \geqslant 0 .
$$

$K(\cdot)$ is uniquely determined as the resolution of the identity for $C$. 
Proof. Let $R(t)$ and $U(t)$ be the positive and the circled parts of $T(t)$ respectively, and let $M>0$ be such that $\left\|E_{t}(\delta)\right\| \leqslant M$ for all $t \geqslant 0$, and all Borel sets $\delta$, where $E_{t}(\cdot)$ is the resolution of the identity for $T(t)$. The resolution of the identity for $U(t)$ has its range contained in that of $E_{t}(\cdot)$ and hence the semigroup $\{U(t)\}$ satisfies all the conditions of Theorem 5.1. Therefore there is a spectral measure $H(\cdot)$ defined on the Borel sets of the real line such that

$$
U(t)=\int_{-\infty}^{\infty} e^{i t \lambda} d H(\lambda)
$$

Moreover $R(t)=\int_{-\infty}^{\omega_{0}} e^{t \mu} d G(\lambda)$, where $G(\cdot)$ is a spectral measure defined on the Borel sets on the real line, $G\left(\left(\omega_{0}, \infty\right)\right)=0$, where $\omega_{0}$ is the type of $\{T(t)\}$. Let $A$ and $i B$ be the infinitesimal generators of $\{R(t)\}$ and $\{U(t)\}$ respectively. $A$ and $B$ are scalar type with resolutions of the identity $G(\cdot)$ and $H(\cdot)$ respectively. Since each $U(s)$ commutes with all $R(t), t \geqslant 0$, Corollary 3.2 implies that $U(s)$ commutes with $G(\alpha)$ for every Borel set $\alpha$. Another application of 3.2 implies that $G(\alpha)$ commutes with $H(\beta)$ for any Borel sets $\alpha$ and $\beta$.

Let $e_{n}=[-n, n]$ and let $X_{n}=G\left(e_{n}\right) H\left(e_{n}\right) X$. Then $X_{n}$ is invariant under $T(t), R(t), U(t)$ and their resolutions of the identity. $\bigcup_{n=1}^{\infty} X_{n}$ is a dense linear manifold in $X$ since $G\left(e_{n}\right)$ and $H\left(e_{n}\right)$ both converge strongly to $I$, and are uniformly bounded. Let $S_{n}$ denote the restriction of $S$ to $X_{n}$ for any operator $S$ in $X$ with $O(S) \supseteq X_{n}$. Therefore $\left\{R_{n}(t)\right\},\left\{U_{n}(t)\right\},\left\{T_{n}(t)\right\}$ are uniformly continuous semigroups of scalar type operators on $X_{n}$ with infinitesimal generators $A_{n}, i B_{n}, C_{n}$ respectively. Moreover $T_{n}(t)=R_{n}(t) U_{n}(t)$, so $C_{n}=A_{n}+i B_{n}$ for all positive integers $n$. It follows from Lemma 5.2 that $C_{n}$ is a scalar type operator. Let $K_{n}(\cdot)$ be its resolution of the identity. Therefore

$$
\begin{aligned}
C_{n} & =\int \lambda d K_{n}(\lambda), & B_{n} & =\int \lambda d H_{n}(\lambda), \\
A_{n} & =\int \lambda d G_{n}(\lambda), & T_{n}(t) & =\int e^{\lambda t} d K_{n}(\lambda) .
\end{aligned}
$$

Hence $A_{n}$ and $B_{n}$ are scalar type with real spectra. Since $C_{n}=A_{n}+i B_{n}$, we get, from [3], that

$$
A_{n}=\int \operatorname{Re} \lambda d K_{n}(\lambda) \text { and } B_{n}=\int \operatorname{Im} \lambda d K_{n}(\lambda) .
$$

It follows that

$$
G_{n}(\alpha)=K_{n}\left(\alpha \times R_{0}\right), \quad H_{n}(\beta)=K_{n}\left(R_{0} \times \beta\right)
$$


where $\alpha, \beta$ are any Borel sets of the real line $R_{0}$. Therefore $K_{n}(\alpha \times \beta)=$ $G_{n}(\alpha) H_{n}(\beta)$. By Lemma 5.2, the range of $K_{n}(\cdot)$ is equal to the range of $E_{n}(t)$ for $t(>0)$ small enough. Hence

$$
\sup \left\{\left\|K_{n}(\pi)\right\|: n=1,2,3, \cdots, \pi \text { a Borel set }\right\} \leqslant M .
$$

For each $\pi \in \Sigma$, define $K(\pi)$ on $\bigcup_{n=1}^{\infty} X_{n}$ by $K(\pi) x=K_{n}(\pi) x, x \in$ $X_{n}$. First we show that this is well defined. Suppose $x \in X_{n} \cap X_{m}$ and $n \leqslant$ m. Then

$$
K_{m}(\alpha \times \beta)=G(\alpha) H(\beta) \mid X_{m}, \quad \text { and } K_{n}(\alpha \times \beta)=G(\alpha) H(\beta) \mid X_{n} .
$$

By standard measure-theoretic results we can show that $X_{n}$ is invariant under $K_{m}$ and $K_{m}(\delta) \mid X_{n}=K_{n}(\delta)$, for any Borel set $\delta$. We will show that $K(\pi)$ extends to a bounded projection, still called $K(\pi)$, that $K(\cdot)$ is a countably additive spectral measure and that $K(\cdot)$ is the tensor product of $G(\cdot)$ and $H(\cdot)$ in the sense that $K(\alpha \times B)=G(\alpha) H(\beta)$ for any Borel sets $\alpha, \beta$ of the real line.

Let $X_{0}=\bigcup_{n=1}^{\infty} X_{n}$. If $\pi$ is any Borel set and $x \in X_{0}$, then $x \in X_{n}$ for some positive integer $n$, and $\|K(\pi) x\|=\left\|K_{n}(\pi) x\right\| \leqslant M\|x\|$. Therefore $K(\pi)$ has a unique extension to a bounded linear operator, still called $K(\pi)$, with $\|K(\pi)\| \leqslant M$. It is routine to see that $K(\pi)$ is a projection, that $K(\pi \cap \delta)=$ $K(\pi) K(\delta)$ and $K(\pi \cup \delta)=K(\pi)+K(\delta)-K(\pi) K(\delta)$ for any Borel sets $\delta, \pi$. One can easily show that $K(\alpha \times \beta)=G(\alpha) H(\beta)$ for any Borel sets $\alpha, \beta$ of the real line. To show that $K(\cdot)$ is countably additive, let $\left\{\pi_{n}\right\}$ be a disjoint collection of Borel sets in the plane and let $\pi=\bigcup_{n=1}^{\infty} \pi_{n}$. If $x \in X_{k}$, then

$$
K(\pi) x=K_{k}(\pi) x=\sum_{n=1}^{\infty} K_{k}\left(\pi_{n}\right) x=\sum_{n=1}^{\infty} K\left(\pi_{n}\right) x .
$$

Therefore

$$
K(\pi) x=\sum_{n=1}^{\infty} K\left(\pi_{n}\right) x, \quad \text { for } x \in X_{0} .
$$

An application of [8, Theorem II 1.18] shows that $\Sigma_{n=1}^{\infty} K\left(\pi_{n}\right)$ converges strongly to a bounded operator which is obviously $K(\pi)$.

Since $G\left(\left(\omega_{0}, \infty\right)\right)=0$, then $K\left(\left(\omega_{0}, \infty\right) \times R_{0}\right)=0$, and hence $\int e^{t \lambda} d K(\lambda)$ is defined and bounded on $X$. But

$$
T(t)\left|X_{n}=\int e^{t \lambda} d K_{n}(\lambda)=\left[\int e^{t \lambda} d K(\lambda)\right]\right| X_{n} .
$$

Hence $T(t)=\int e^{t \lambda} d K(\lambda), t \geqslant 0$. It follows, by Theorem 2.1, that the infinitesimal generator $C$ is a scalar type operator with resolution of the identity $K(\cdot)$. 
The uniqueness of $K(\cdot)$ follows also from 2.1. This ends the proof of the theorem.

REMARK. The problem can be easily reduced to the Hilbert space case if we assume that $G(\cdot)$ and $H(\cdot)$ generate a bounded Boolean algebra of projections $E$. This is the case if in particular we assume that the resolutions of the identity, $E_{t}(\cdot)$, generate a bounded Boolean algebra of projections, or if $X$ is one of the spaces $L_{p}(1<p<\infty)$ since in such space any two commuting bounded Boolean algebras of projections generate a bounded Boolean algebra of projections as shown by McCarthy [17]. Let $A$ and $W$ be the algebras generated by $E$ in the uniform and weak topologies respectively. $X$ can be equivalently renormed so as to make all the operators in $E$ hermitian as shown by Berkson [3]. We suppose such a renorming is carried out. A becomes a commutative $V^{*}$-algebra, and hence $W$, the weak closure of $A$, is a commutative $V^{*}$-algebra as shown by Palmer [18, Corollary 2.9]. It follows (see [5, Theorems $3.1,3.2]$ ) that there is an isometric *-representation $\phi$ of $W$ onto a von Neumann algebra of operators on a Hilbert space $Y$, and the restriction of $\phi$ to any bounded subset of $W$ is bicontinuous in the weak-weak and the strong-strong operator topologies. Now $\phi(G(\cdot))$ and $\phi(H(\cdot))$ are two commuting spectral measures with values in $B(Y)$, and hence can be "amalgamated" in the manner of Berberian [1, §11]. If $F(\cdot)$ is their "amalgam," then $\phi^{-1}(F(\cdot))$ gives the required spectral measure $K(\cdot)$.

(5.4) Corollary. An operator $A$ in a weakly complete Banach space $X$ generates a strongly continuous semigroup of scalar type operators with uniformly bounded resolutions of the identity if and only if $A$ is scalar type and $\sigma(A)$ lies in some left half plane $\{\lambda: \operatorname{Re} \lambda \leqslant \omega\}$.

REMARK. We cannot replace "scalar type" by "spectral" in Theorem 5.3. The following example shows that there are strongly continuous semigroups of spectral operators with resolutions of the identity uniformly bounded (or even generate a bounded Boolean algebra), but the infinitesimal generator is not spectral, even when the underlying space is a Hilbert space.

(5.5) ExAmple. Let $X=L_{2}[0,1]$, and for any $x \in X, t \geqslant 0$, let

$$
(T(t) x)(s)= \begin{cases}x(t+s), & t+s \leqslant 1 \\ 0, & t+s>1\end{cases}
$$

Therefore $T(t)=0$ for $t \geqslant 1$, and $\{T(t)\}$ is a semigroup of operators, nilpotent for $t>0$, hence spectral for $t \geqslant 0$. The resolutions of the identity for all the operators $T(t)(t>0)$ are identical and trivial, namely $E_{t}(\delta)=I$ or 0 according to whether $0 \in \delta$ or not. The semigroup is strongly continuous, for if $x$ is a continuous function on $[0,1]$ (and hence uniformly continuous), 
and $0 \leqslant t \leqslant 1$, then

$$
\|T(t) x-x\|^{2}=\int_{0}^{1-t}|x(s+t)-x(s)|^{2} d s+\int_{1-t}^{1}|x(s)|^{2} d s .
$$

The first integral converges to 0 as $t \rightarrow 0$ by the uniform continuity of $x$; the second integral is $\leqslant t$ sup $\{|x(s)|: 0 \leqslant s \leqslant 1\}$, hence converges to 0 as $t \rightarrow 0$. Hence $T(t) x \rightarrow x$, as $t \rightarrow 0$, for $x$ continuous. But the continuous functions are dense in $L_{2}[0,1]$, and $\{T(t)\}$ is uniformly bounded. Therefore $T(t) x \rightarrow x$, as $t \rightarrow 0$, for all $x \in L_{2}[0,1]$ by [8, Theorem II 1.18]. Thus the semigroup is strongly continuous at 0 , and hence strongly continuous everywhere (see [12, Theorem 10.5.5]).

Since $T(t)=0$ for $t \geqslant 1$, we have

$$
\lim _{t \rightarrow \infty} \frac{\log \|T(t)\|}{t}=-\infty \text {. }
$$

Therefore, the semigroup is of type $-\infty$, and the infinitesimal generator has empty spectrum. The generator cannot be spectral since a spectral operator has nonempty spectrum (see [8, XVIII 2.1 and 2.2]).

6. Normal operators on Banach spaces and a theorem of Foiaş. In [11], Foias proved that any strongly continuous semigroup $\{T(t)\}$ of scalar type operators on a Hilbert space, having their resolutions of the identity uniformly bounded, is similar to a semigroup $\{N(t)\}$ of normal operators. We give a similar result for weakly complete Banach spaces and show that it reduces to Foiaş' result in Hilbert spaces. First we define a normal operator on a Banach space.

(6.1) Definition. A bounded operator on a Banach space $X$ is said to be normal if it is a scalar type operator with all the projections in the range of its resolution of the identity hermitian.

This definition differs from Palmer's [18], but is equivalent to it in weakly complete spaces, and in general any operator normal in the sense of 6.1 is normal in the sense of Palmer (see [18, Theorem 5.1]). According to his definition a bounded operator $T$ is normal if and only if $T=R+i J$, where $R, J$ are hermitian, and $\left\{e^{i t R}:-\infty<t<\infty\right\} \cup\left\{e^{i t J}:-\infty<t<\infty\right\}$ is contained in a commutative $V^{*}$-algebra.

(6.2) THEOREM. Let $\{T(t)\}$ be a strongly continuous semigroup of scalar type operators on a weakly complete Banach space $X$, with their resolutions of the identity uniformly bounded in norm. Then $X$ can be equivalently renormed so that every $T(t)$ becomes normal. Moreover, if $X$ is given as a Hilbert space, the new norm can be chosen to be a Hilbert space norm.

Proof. From Theorem 5.3, we get $T(t)=\int e^{\lambda t} d K(\lambda)$, where $K(\cdot)$ is a strongly countably additive spectral measure. Therefore $X$ can be renormed so 
as to make $K(\delta)$ hermitian for every Borel set $\delta$ [3, Lemma 2.3]. If $X$ is a Hilbert space, the new norm can be chosen to be a Hilbert space norm [15, Theorem 6]. Since the range of the resolution of the identity for $T(t)$, for any $t \geqslant 0$, is contained in the range of $K(\cdot)$, the result follows.

(6.3) Corollary (Foiaş' TheOREM). If $\{T(t)\}$ is as in Theorem 6.2, and if $X$ is a Hilbert space, then $\{T(t)\}$ is similar to a semigroup $\{N(t)\}$ of normal operators, i.e., there exists a regular positive selfadjoint $T \in B(X)$ such that $T(t)=A^{-1} N(t) A$.

Proof. Let $|\cdot|$ be the new Hilbert space norm under which the projections $K(\delta)$ are hermitian, and let $\langle\cdot, \cdot\rangle$ be the inner product compatible with this norm. The original norm and inner product are denoted by $\|\cdot\|$ and $(\cdot, \cdot)$ respectively. Let $B$ be the bounded operator defined by $(B x, y)=\langle x, y\rangle$. Therefore $B$ is positive selfadjoint in the original norm, for the numerical range of $B$ is $W(B)=\{(B x, x):\|x\|=1\}=\left\{|x|^{2}:\|x\|=1\right\}$, which is positive. It is also obvious that $B$ is invertible. Let $A$ be the positive square root of $B$, hence $\langle x, y\rangle=(A x, A y)$.

If $\delta$ is any Borel set, then $A K(\delta) A^{-1}$ is selfadjoint in the original norm since its numerical range is given by

$$
\begin{aligned}
W\left(A K(\delta) A^{-1}\right) & =\left\{\left(A K(\delta) A^{-1} x, x\right):\|x\|=1\right\} \\
& =\left\{\left\langle K(\delta) A^{-1} x, K(\delta) A^{-1} x\right\rangle:\left|A^{-1} x\right|=1\right\},
\end{aligned}
$$

which is the (necessarily nonnegative) numerical range of $K(\delta)$ with respect to the new norm. Let $N(t)=A T(t) A^{-1}$, therefore $N(t)=\int e^{\lambda t} d\left(A K(\lambda) A^{-1}\right)$ which is obviously normal.

\section{A perturbation theorem.}

(7.1) THEOREM. Let $\{T(t)\}$ be a strongly continuous semigroup of scalar type operators on a weakly complete Banach space $X$, with the operators $T(t)$ having their resolutions of the identity $E_{t}(\cdot)$ uniformly bounded in norm by $M>0$. Then $f(T(t)) \rightarrow f\left(T\left(t_{0}\right)\right)$ strongly, as $t \rightarrow t_{0}$, for every bounded Borel function $f$ such that the closure of its set of discontinuities is an $E_{t_{0}}(\cdot)$ null set.

Proof. Let $x \in X$, and let $f$ be a function satisfying the condition above. The result to be proven amounts to showing that the function $t \rightarrow$ $f(T(t)) x$ is continuous at $t_{0}$. Since this is a function between metric spaces, it suffices to prove that $f\left(T\left(t_{n}\right)\right) x \rightarrow f\left(T\left(t_{0}\right)\right) x$ whenever the sequence $\left\{t_{n}\right\}$ converges to $t_{0}$.

First we prove the result for continuous functions $f$. By the principle of uniform boundedness, there is a $K>0$ such that $\left\|T\left(t_{0}\right)\right\| \leqslant K$, and $\left\|T\left(t_{n}\right)\right\| \leqslant K$, 
for all $n$, and hence all the spectra $\sigma\left(T\left(t_{n}\right)\right)$ and $\sigma\left(T\left(t_{0}\right)\right)$ are contained in the disk $D=\{\lambda:|\lambda| \leqslant K\}$. Let $K(\cdot)$ be the resolution of the identity for the generator $C$ of $\{T(t)\}$. Then there is a half plane $\pi=\left\{\lambda: \operatorname{Re} \lambda \leqslant \omega_{0}\right\}$ containing $D$, such that $K(\pi)=I$. Let $g$ be a bounded continuous extension of $f \mid D$ to all of the complex plane. $g\left(\exp t_{n} \lambda\right) \rightarrow g\left(\exp t_{0} \lambda\right)$ pointwise for $\lambda \in$ $\pi$. Since $g$ is bounded, the bounded convergence theorem for vector measures gives

$$
\int g\left(\exp t_{n} \lambda\right) d K(\lambda) x \rightarrow \int g\left(\exp t_{0} \lambda\right) d K(\lambda) x, \quad x \in X .
$$

Comparison of the resolutions of the identity now shows that

$$
\int g\left(\exp t_{n} \lambda\right) d K(\lambda)=g\left(T\left(t_{n}\right)\right)=f\left(T\left(t_{n}\right)\right)
$$

for $n=0,1,2, \cdots$. This proves the theorem for continuous $f$.

The proof of the theorem in the general case can now be based on the argument of [8, Theorem XVII 4.3].

(7.2) COROLlaRY. With the same notation as above, if $E_{t_{0}}(\partial \alpha)=0$, then $E_{t_{0}}(\alpha) x=\lim _{t \rightarrow t_{0}} E_{t}(\alpha) x$ for every $x \in X$.

(7.3) Corollary. With the same notation as above, the functions $t \rightarrow$ $\operatorname{Re} T(t)$, and $t \rightarrow \operatorname{Im} T(t)$ are strongly continuous.

8. Semigroups of hermitian operators. We show that a strongly continuous semigroup of hermitian operators on a Banach space $X$ has a self-conjugate generator $\boldsymbol{A}$ (see definitions below). This generalizes the Hille-Sz.-Nagy theorem. We follow Palmer [18] in making the following definitions.

(8.1) Definition. An operator $R$ is said to be self-conjugate if $i R$ generates a strongly continuous group of isometries.

This definition agrees with the usual definition of selfadjoint operators on a Hilbert space via the well-known theorem of M. H. Stone on unitary groups (see [12, p. 598] or [19]). Also in the case $R$ is bounded, $R$ is self-conjugate in the above sense if and only if $R$ is hermitian (see [6]).

(8.2) Definition. An operator $R$ is said to be symmetric if the set

$$
\left\{x^{*} R x: x \in O(R), x^{*} \in X^{*},\left\|x^{*}\right\|=\|x\|=x^{*}(x)=1\right\}
$$

is a subset of the real line.

This definition agrees with the usual one on a Hilbert space.

(8.3) THEOREM. If $\{T(t)\}$ is a strongly continuous semigroup of hermitian operators on $X$, then the infinitesimal generator $A$ is self-conjugate. In particular $A$ is maximal symmetric. 
Proof. We start by showing that $A$ is symmetric. Let $x \in D(A), x^{*} \in$ $X^{*}$ with $\left\|x^{*}\right\|=\|x\|=x^{*}(x)=1$. Then $x^{*} T(t) x$ is real for every $t \geqslant 0$, and hence $x^{*} A x=\lim _{t \rightarrow 0}\left(x^{*} T(t) x-1\right) / t$ is real, i.e., $A$ is symmetric. Since $A$ is also closed and densely defined, it follows from [18, Lemmas 3.1, 3.2] that $A$ is either self-conjugate or else its residual spectrum contains at least one of the nonreal half planes. But $\sigma(A)$ is contained in some left half plane $\left\{\lambda: \operatorname{Re} \lambda \leqslant \omega_{0}\right\}$, thus $A$ is self-conjugate. It follows again from [18, Lemma 3.2] that $A$ is maximal symmetric. This ends the proof of the theorem.

If $X$ is weakly complete, then a strongly continuous semigroup of hermitian operators consists of scalar type operators.

(8.4) THEOREM. Let $\{T(t)\}$ be a strongly continuous semigroup of operators on a weakly complete Banach space $X$. Then $\{T(t)\}$ is a hermitian-equivalent family if and only if every $T(t)$ is scalar type with real spectrum.

Proof. The "if" part is established in Corollary 3.3 and is valid in arbitrary Banach spaces. To prove the "only if" part we notice that for any $t>0$, the operators $T(t)^{n}, n=0,1,2, \cdots$, can be made simultaneously hermitian since $(T(t))^{n}=T(n t)$. Hence $T(t)$ is scalar type by [3].

REMARK. The "only if" is not valid in all Banach spaces as shown by the following example.

(8.5) EXAMPLE. Let $X=l_{\infty}$, and for each $t \geqslant 0$, let $T(t)$ be the operator represented by the diagonal matrix $\operatorname{diag}\left(e^{t}, e^{t / 2}, e^{t / 3}, \cdots\right)$. Thus $\{T(t)\}$ is a uniformly continuous semigroup with generator $A=$ $\operatorname{diag}(1,1 / 2,1 / 3, \cdots)$. Each $T(t)$ is hermitian since if $r$ is a real number, then

$$
\|I+\operatorname{ir} T(t)\|=\left|1+\operatorname{ire}^{t}\right|=1+o(r) \quad \text { as } r \rightarrow 0 .
$$

However, no $T(t)$, except $T(0)=I$, is spectral (see [9]).

REMARK. Unlike the Hilbert space case, there is a self-conjugate operator $A$ on a reflexive Banach space $X$ that generates a semigroup which does not consist entirely of hermitian operators. Indeed, if $A$ is any bounded hermitian operator which is not scalar type on a reflexive space $X$ (see [14]), then $A$ generates the semigroup $\left\{e^{t A}: t \geqslant 0\right\}$. If $e^{t A}$ is hermitian for every $t \geqslant 0$, then $e^{t A}$ is scalar type for every $t \geqslant 0$ by Theorem 8.4 , and $A$ is scalar type, a contradiction.

9. Semigroups of unbounded operators. In [7] Devinatz extended the Hille-Sz.-Nagy theorem to semigroups of unbounded selfadjoint operators on a Hilbert space. Here we extend this result to semigroups of unbounded scalar type operators with real spectra on a Banach space. This gives an unbounded version of Theorem 3.1. 
(9.1) THEOREM. Let $\{T(t): t>0\}$ be a family of scalar type operators (possibly unbounded) with spectra on the real line. Suppose $\{T(t)\}$ satisfies the following conditions.

(i) $T(t+s) \subseteq T(t) T(s), t, s>0$;

(ii) For every $x \in \bigcap_{t>0} D(T(t))$ and every $x^{*} \in X^{*}$, the function $t \rightarrow x^{*} T(t) x$ is continuous.

Then there exists a unique spectral measure $E(\cdot)$ on the Borel sets of $[0, \infty)$ sach that $T(t)=\int \lambda^{t} d E(\lambda)$. Moreover, $\bigcap_{t>0} D(T(t))$ is dense in $X$. There is a projection $P \in B(X)$ such that $T(t) x \rightarrow P x$ (as $t \rightarrow 0$ ), for every $x \in \bigcap_{t>0} D(T(t))$.

Proof. Let $E(\cdot)$ be the resolution of the identity for $T(1)$ and $F(\cdot)$ be that for $T(1 / 2)$. It follows that

$$
T(1) \subseteq(T(1 / 2))^{2}=\int_{-\infty}^{\infty} \lambda^{2} d F(\lambda) .
$$

But $T(1)$ is scalar type; so we must have equality. This implies that $\sigma(T(1))$ is nonnegative, and a similar argument shows that $\sigma(T(t))$ is nonnegative, for every $t \geqslant 0$. Also $E(\delta)=F(\sqrt{\delta})$, or equivalently $F(\alpha)=E\left(\alpha^{2}\right)$, where $\delta$ and $\alpha$ are any Borel sets included in $[0, \infty)$. Therefore

$$
T(1 / 2)=\int_{0}^{\infty} \lambda^{1 / 2} d E(\lambda)
$$

since the operator defined by the integral is scalar type whose resolution of the identity agrees with that of $T(1 / 2)$. Similarly

$$
T(1 / n)=\int_{0}^{\infty} \lambda^{1 / n} d E(\lambda)
$$

It follows from property (i) that

$$
T(m / n) \subseteq(T(1 / n))^{m}=\int_{0}^{\infty} \lambda^{m / n} d E(\lambda)
$$

Again we must have equality.

Let $S(t)=\int_{0}^{\infty} \lambda^{t} d E(\lambda)$; then we have proved that $T(t)=S(t)$ for all rational $t>0$.

Let $e_{n}=[0, n], X_{n}=E\left(e_{n}\right) X, X_{\infty}=\bigcup_{n=1}^{\infty} X_{n}$. Hence $X_{\infty}$ is dense in $X$, every $X_{n}$ is invariant under $S(t)$, for $t>0$, and $S(t) \mid X_{n}$ is bounded, for $t>0$. To prove the same for $T(t)$, let $t>0$ and choose a rational number $s>t$, and $x \in X_{n}$. Hence

$$
x \in D(S(s))=D(T(s)) \subseteq D(T(s-t) T(t)) \subseteq D(T(t)) .
$$

Moreover if $x^{*} \in X^{*}$ belongs to the annihilator of $X_{n}$, i.e., $x^{*}\left(X_{n}\right)=0$, then 


$$
\begin{aligned}
x^{*} T(t) x & =\lim \left\{x^{*} T\left(s_{n}\right) x: s_{n} \text { rational, } s_{n} \rightarrow t\right\} \\
& =\lim \left\{x^{*} S\left(s_{n}\right) x: s_{n} \text { rational, } s_{n} \rightarrow t\right\}=0 .
\end{aligned}
$$

By the Hahn-Banach theorem, $T(t) x \in X_{n}$, i.e., $X_{n}$ is invariant under $T(t)$. Moreover $T(t) \mid X_{n}$ is closed, everywhere defined, and hence is bounded by the closed graph theorem. To show that $T(t)\left|X_{\infty}=S(t)\right| X_{\infty}$, let $x \in X_{\infty}$, hence $x \in X_{n}$ for some positive integer $n$, and let $\left\{t_{k}\right\}$ be a sequence of rational numbers with $t_{k} \rightarrow t$. Therefore

$$
\begin{aligned}
x^{*} T(t) x & =\lim _{k \rightarrow \infty} x^{*} T\left(t_{k}\right) x \\
& =\lim _{k \rightarrow \infty} x^{*} S\left(t_{k}\right) x=x^{*} S(t) x, \quad x^{*} \in X^{*} .
\end{aligned}
$$

Therefore $T(t) x=S(t) x$. To prove that $T(t)=S(t)$, first suppose that $x \in$ $O(S(t))$, then $E\left(e_{n}\right) x \rightarrow x$, and

$$
\begin{aligned}
T(t) E\left(e_{n}\right) x & =S(t) E\left(e_{n}\right) x=\int_{0}^{n} \lambda^{t} d E(\lambda) x \\
& \rightarrow \int_{0}^{\infty} \lambda^{t} d E(\lambda) x=S(t) x
\end{aligned}
$$

Since $T(t)$ is closed, it follows that $x \in D(T(t))$, and $T(t) x=S(t) x$; thus $T(t) \supseteq S(t)$. But since both $T(t)$ and $S(t)$ are scalar type operators, we must have $T(t)=S(t)$.

The uniqueness of $E(\cdot)$ follows from the fact that if $T(t)=\int_{0}^{\infty} \lambda^{t} d G(\lambda)$, then $G(\cdot)$ is necessarily the resolution of the identity for $T(1)$.

$\bigcap_{t>0} D(T(t))$ is dense in $X$ since it obviously contains $X_{\infty}$.

Let $P=E((0, \infty))=I-E(0)$. If $x \in \bigcap D(T(t)),\left\{t_{n}\right\}$ is a sequence of positive numbers converging to 0 , and $k$ denotes the characteristic function of $(0, \infty)$, then

$$
T\left(t_{n}\right) x=\int \lambda^{t} d E(\lambda) x \rightarrow \int k(\lambda) d E(\lambda) x=P x .
$$

Therefore $\lim _{t \rightarrow 0} T(t) x=P x$.

10. Circled representations of locally compact abelian groups. In what follows we extend the Godement-Naimark theorem about unitary representations of LCA (i.e., locally compact abelian) groups (see [13]). Here we assume that the resolutions of the identity of all the operators generate a bounded Boolean algebra of projections. It is conceivable that the theorem is true under weaker assumptions, e.g., that the resolutions of the identity are only uniformly bounded.

(10.1) THEOREM. Let $G$ be an LCA group and $s \rightarrow U(s)$ be a representation of $G$ in $B(X)$, where $X$ is weakly complete, and each $U(s)$ is a 
circled scalar type operator with resolution of the identity $E_{s}(\cdot)$. Assume that the projections $E_{s}(\delta)$, for $s \in G, \delta$ Borel set, generate a bounded Boolean algebra of projections $E$ Then there exists a unique countably additive spectral measure $F(\cdot)$ defined on the Borel sets of $\hat{G}$, the dual group of $G$, such that

$$
U(s)=\int_{\hat{G}} \overline{(s, \gamma)} d F(\gamma), \quad s \in G .
$$

Proof. A proof could be given by modifying the proof of the GodementNaimark theorem (see [13]), i.e., by basing the demonstration on an induced representation of the Banach algebra $L^{1}(G)$. Instead we shall show how the problem can be reduced to the Hilbert space case.

Let $A$ and $W$ be the uniformly closed and weakly closed algebras of operators generated by $E$ respectively. Assume $X$ is renormed (by an equivalent norm) so that all the operators in $E$ are hermitian. Then $A$ becomes a commutative $V^{*}$-algebra, and hence, by [18, Corollary 2.9], " $W$ is a $V^{*}$-algebra. It follows (see [5]) that there is a ${ }^{*}$-isomorphism $\phi$ of $W$ onto a von Neumann algebra of operators on a Hilbert space $H$, with $\phi$ bicontinuous on bounded sets if both $B(X)$ and $B(H)$ are given the strong operator topology or the weak operator topology. Therefore $s \rightarrow \phi(U(s))$ is a unitary representation of $G$, and by the Godement-Naimark theorem there is a countably additive spectral measure $K(\cdot)$ defined on the Borel sets of $\hat{G}$ and taking values in the hermitian projections of $\phi(\omega)$ such that

$$
\phi(U(s))=\int_{\hat{G}}(\overline{s, \gamma}) d K(\gamma), \quad s \in G .
$$

Let $F(\cdot)=\phi^{-1}(K(\cdot))$, then $F(\cdot)$ is a countably additive spectral measure defined on the Borel sets of $G$ since $\phi^{-1}$ is strongly continuous on bounded sets. Therefore

$$
U(s)=\int_{\hat{G}}(\overline{s, \gamma}) d F(\gamma), \quad s \in G .
$$

The uniqueness of $F(\cdot)$ follows from the fact that the Fourier-Stieltjes transform of a (scalar-valued) measure determines the measure uniquely. Precisely if $x \in X, x^{*} \in X^{*}$, and $F_{1}(\cdot)$ is any spectral measure satisfying the desired conditions, then

$$
\int(\overline{s, \gamma}) d\left(x^{*} F_{1}(\gamma) x\right)=x^{*} U(s) x=\int \overline{(s, \gamma)} d\left(x^{*} F(\gamma) x\right),
$$

for all $s \in G$. Hence $x^{*} F_{1}(\delta) x=x^{*} F(\delta) x$, for every Borel set $\delta \subseteq \hat{G}$ and every $x \in X, x^{*} \in X^{*}$. Therefore $F_{1}(\cdot)=F(\cdot)$.

For the next result we need the following lemma which is probably well known but no proof of which seems to exist in the literature. It is the converse of a standard result. 
(10.2) LemmA. Let $K$ be a subset of an LCA group $G$, and let $\left(x, \gamma_{\alpha}\right) \rightarrow 1$ uniformly on $K$ for every net $\left\{\gamma_{\alpha}\right\}$ which converges to 0 in $\hat{G}$. Then $\bar{K}$ is compact.

Proof. Given $\epsilon>0$, there exists a neighborhood $V$ of 0 in $\hat{G}$ such that $|(x, \gamma)-1|<\epsilon$, for all $x \in K, \gamma \in V$. We can assume that $V$ has a finite Haar measure, for otherwise we can replace $V$ by its intersection with a neighborhood of 0 of finite measure. Let $f=k_{V}$, the characteristic function of $V$. Then $f \in L^{1}(\hat{G})$, and

$$
\begin{aligned}
|\hat{f}(x)-\mu(V)| & =\left|\int_{\hat{G}}(\overline{x, \gamma}) f(\gamma) d \gamma-\int_{\hat{G}} f(\gamma) d \gamma\right| \\
& \leqslant \int_{V}|\overline{(x, \gamma)}-1| d \gamma \leqslant \epsilon \mu(V), \quad x \in K,
\end{aligned}
$$

where $\mu$ is the Haar measure on $\hat{G}$. Hence $|\hat{f}(x)| \geqslant(1-\epsilon) \mu(V)$, for all $x \in$ $K$. But $\mu(V)>0$ and $\hat{f}$ vanishes at infinity; therefore $\bar{K}$ is compact.

(10.3) COROLlaRY. With the same notation as in (10.1), the representation $s \rightarrow U(s)$ is uniformly continuous if and only if $F(\cdot)$ is supported by a compact subset of $\hat{G}$.

Proof. Let $K=\operatorname{supp} F(\cdot)$. If $K$ is compact, then $(s, \gamma) \rightarrow\left(s_{0}, \gamma\right)$ uniformly on $K$ as $s \rightarrow s_{0}$. It follows that

$$
\int(\overline{s, \gamma}) d F(\gamma) \rightarrow \int \overline{\left(s_{0}, \gamma\right)} d F(\gamma)
$$

uniformly as $s \rightarrow s_{0}$. Conversely if the representation is uniformly continuous, then for every $\epsilon>0$, there exists a neighborhood $V$ of 0 in $G$ such that $\|U(s)-I\|<\epsilon$, for all $s \in V$. But

$$
U(s)-I=\int[\overline{(s, \gamma)}-1] d F(\gamma),
$$

hence

$$
\sup _{\gamma \in K}|(s, \gamma)-1|=\|U(s)-I\|_{\mathrm{sp}} \leqslant\|U(s)-I\|<\epsilon, \quad s \in V,
$$

where $\|\cdot\|_{s p}$ denotes the spectral radius. Therefore $(s, \gamma)$ is continuous in $s$, uniformly for $\gamma \in K$, and so $K=\bar{K}$ is compact.

11. Operational calculus. An operational calculus for infinitesimal generators $A$ of strongly continuous semigroups was developed by Hille and Phillips (see [12, Chapter XV]). This was defined for a certain class of functions $f$ such that $f(A)$ is always bounded. Balakrishnan [2] extends the calculus to a bigger class of functions and allows $f(A)$ to be unbounded. This calculus also gives a 
definition for certain fractional powers of $A$ which were developed independently by Yosida and others (see [21]). These two definitions of fractional powers are equivalent.

On the other hand, there is a natural operational calculus for (not necessarily bounded) scalar type operators developed by Bade (see [8, Chapter XVIII]). If $A=\int \lambda d E(\lambda)$, then $f(A)$ is defined by $f(A)=\int f(\lambda) d E(\lambda)$, for any Borel measurable function $f$.

It is the purpose of this section to show that the two calculi coincide for scalar type operators $A$ whose spectrum is included in a left half plane or, equivalently, generate a strongly continuous semigroup.

First we give a very brief description of the Hille-Phillips-Balakrishnan calculus. Let $\{T(t)\}$ be a strongly continuous semigroup of type $\omega_{0}$ with infinitesimal generator $A$. It is desired that $f_{t}(A)$ should be $T(t)$, for $f_{t}(\lambda)=e^{t \lambda}$. Accordingly, if $\phi$ is the Laplace-Stieltjes transform $\hat{\mu}$ of a measure $\mu$, i.e., if $\phi(\lambda)=\hat{\mu}(\lambda)=\int_{0}^{\infty} e^{\lambda t} d \mu(t)$, then $\phi(A)$ is defined by

$$
\phi(A)=\int_{0}^{\infty} T(t) d \mu(t) .
$$

This definition makes sense for the class $S$ of countably additive measures on the Borel sets of $[0, \infty)$ for which

$$
\|\mu\|=\int_{0}^{\infty}\|T(t)\| d|\mu|(t)<\infty .
$$

With $\|\cdot\|$ as a norm, and convolution as multiplication, $S$ becomes a Banach algebra. Hille and Phillips extended this calculus to a class of functions, larger than $\{\hat{\mu}: \mu \in S\}$, but we will not discuss this extension here.

To explain Balakrishnan's calculus, let $L$ be the closed ideal of $S$ consisting of measures which are absolutely continuous with respect to Lebesgue measure. We shall not distinguish between measures in $L$ and their RadonNikodym derivatives (with respect to Lebesgue measure), as this does not lead to confusion. If $\phi$ is a Borel function, let $C_{\phi}$ be the operator defined by

$$
\begin{aligned}
\partial\left(C_{\phi}\right) & =\{f \in L: \phi \hat{f}=\hat{g} \text { for some } g \in L\}, \\
C_{\phi} f & =g, \text { where } \phi \hat{f}=\hat{g} .
\end{aligned}
$$

Let $M$ be the set of all Borel functions $\phi$ for which $D\left(C_{\phi}\right)$ is dense in $L$. For every $\phi \in M$, the operator $\phi(A)$ is defined as follows. First define $T(\phi ; A)$ by the equations

$$
\begin{aligned}
\mathcal{D}(T(\phi ; A)) & =\left\{\hat{f}(A) x: f \in \mathcal{D}\left(C_{\phi}\right), x \in X\right\}, \\
T(\phi ; A) \hat{f}(A) x & =\hat{g}(A) x \quad \text { if } \phi \hat{f}=\hat{g}, x \in X .
\end{aligned}
$$


$T(\phi ; A)$ can be shown to be well defined. $\phi(A)$ is, then, defined to be the smallest closed extension of $T(\phi ; A)$. It follows that $\phi(A)$ is a closed operator, and is bounded if and only if $\phi=\hat{\mu}$ for some $\mu \in S$, and if this is the case, the definition of $\phi(A)$ agrees with the Hille-Phillips definition. So the Balakrishnan calculus extends that of Hille-Phillips. The algebra $M$ contains all polynomials and certain fractional powers.

If $A$ is a scalar type operator with $\sigma(A)$ contained in a left half plane, and resolution of the identity $E(\cdot)$, then $A$ generates a strongly continuous semigroup $\{T(t)\}$, and the Balakrishnan calculus gives a definition for $\phi(A)$, for $\phi \in M$. Moreover, the Bade calculus defines $\phi(A)$ to be $\int \phi(\lambda) d E(\lambda)$.

(11.1) Theorem. Let $A$ be a scalar type operator with $\sigma(A)$ contained in a left half plane $\left\{\lambda: \operatorname{Re} \lambda \leqslant \omega_{0}\right\}$, and resolution of the identity $E(\cdot)$. Then the Balakrishnan and the Bade operational calculi agree on $M$.

Proof. $\phi(A)$ will always denote the operator given by the Balakrishnan calculus. First we consider the case $\phi=\hat{\mu}$, with $\mu \in S$. Then

$$
\begin{aligned}
x^{*} \phi(A) x & =\int_{0}^{\infty} x^{*} T(t) x d \mu(t) \\
& =\int_{0}^{\infty} \int_{\sigma(A)} e^{t \lambda} d\left(x^{*} E(\lambda) x\right) d \mu(t), \quad x \in X, x^{*} \in X^{*} .
\end{aligned}
$$

Fubini's theorem can be applied to interchange the order of integration since $e^{t \lambda}$ is integrable with respect to the measure $\left|x^{*} E(\cdot) x\right| \times|\mu|$. We have then

$$
x^{*} \phi(A) x=\int_{\sigma(A)} \phi(\lambda) d\left(x^{*} E(\lambda) x\right)
$$

and

$$
\phi(A)=\int_{\sigma(A)} \phi(\lambda) d E(\lambda) .
$$

Now let $\phi \in M$, and set $B=\int \phi(\lambda) d E(\lambda)$. Let $z \in D(T(\phi ; A))$. There exist $x \in X$, and $f$ and $g \in L$ such that $z=\hat{f}(A) x$, and $\phi \hat{f}=\hat{g}$.

$$
\begin{aligned}
T(\phi ; A) z & =\hat{g}(A) x=\int \hat{g}(\lambda) d E(\lambda) x \\
& =\int \phi(\lambda) \hat{f}(\lambda) d E(\lambda) x .
\end{aligned}
$$

Therefore $B z$ exists and $B z=T(\phi ; A) y$ (see [8, XVIII 2.11]), i.e., $T(\phi ; A) \subseteq$ $B$, and hence $\phi(A) \subseteq B$.

We will show that $D(T(\phi ; A))$ contains $E(\delta) X$, for every compact set $\delta$. For every point $\lambda_{0}$ with $\operatorname{Re} \lambda_{0} \leqslant \omega_{0}$ we can find $f_{0} \in D\left(C_{\phi}\right)$ with $\hat{f}_{0}\left(\lambda_{0}\right) \neq$ 0 , for otherwise $\hat{f}\left(\lambda_{0}\right)=0$ for all $f \in D\left(C_{\phi}\right)$ which is dense in $L$, and hence 
$\hat{f}\left(\lambda_{0}\right)=0$ for all $f \in L$. Let $g \in L_{1}[0, \infty)$, and set $f(t)=e^{-t \omega_{0}} g(t)$, then $f \in L$, due to the fact that $e^{-t \omega_{0}}\|T(t)\|$ is a bounded function of $t$. Therefore $\hat{g}\left(\lambda_{0}-\omega_{0}\right)=\hat{f}\left(\lambda_{0}\right)=0$, for all $g \in L_{1}[0, \infty)$, which is impossible. Thus, for every $\lambda_{0}$ with $\operatorname{Re} \lambda_{0} \leqslant \omega_{0}$, there is an $f_{0} \in L$ with $\hat{f}_{0}\left(\lambda_{0}\right) \neq 0$, and hence $\hat{f}_{0}$ never vanishes on a suitable disk $\delta_{0}=\left\{\lambda:\left|\lambda-\lambda_{0}\right| \leqslant \epsilon_{0}\right\}$. Choose $h_{0} \in L$ such that $\left\|h_{0}-f_{0}\right\|$ is small enough so that $\hat{h}_{0}$ never vanishes on $\delta_{0}$ and $h_{0} \in D\left(C_{\phi}\right)$; this is possible since $D\left(C_{\phi}\right)$ is dense in $L$. If $x \in E\left(\delta_{0}\right) X$, then $x=\hat{h}_{0}(A) y$, where $y=\int_{\delta_{0}}\left(\hat{h}_{0}(\lambda)\right)^{-1} d E(\lambda) x$, i.e., $E\left(\delta_{0}\right) X \subseteq O(T(\phi ; A))$. If $\delta$ is any compact set, it can be covered by a finite number of disks with the property above; hence

$$
E(\delta) X \subseteq D(T(\phi ; A)) \subseteq D(\phi(A))
$$

To prove that $B=\phi(A)$, let $x \in D(B)$, and put $x_{n}=E\left(e_{n}\right) x$, where $e_{n}=\{\lambda:|\lambda| \leqslant n\}$. Then $x_{n} \in D(T(\phi ; A)), x_{n} \rightarrow x$, and $\phi(A) x_{n}=B x_{n} \rightarrow B x$. Therefore $x \in \mathcal{D}(\phi(A))$ and $\phi(A) x=B x$, i.e., $B \subseteq \phi(A)$. Hence $B=\phi(A)$. This ends the proof of the theorem.

Fractional powers of an operator $(-A)$ such that $A$ generates a strongly continuous uniformly bounded semigroup were defined and developed by Phillips, Balakrishnan, and Yosida (see [21, IX 11]). If $0<\alpha<1$, then $(-A)^{\alpha}$ is defined to be the negative of the generator of the semigroup $\{\hat{T}(t, \alpha): t \geqslant 0\}$ given by

$$
\begin{aligned}
\hat{T}(t, \alpha) x & =\int_{0}^{\infty} f_{t, \alpha}(s) T(s) x d s, \text { for } t>0, \\
\hat{T}(0, \alpha) & =I,
\end{aligned}
$$

where

$$
f_{t, \alpha}(\lambda)=\left\{\begin{array}{l}
\frac{1}{2 \pi i} \int_{\sigma-i \infty}^{\sigma+i \infty} \exp \left(z \lambda-t z^{\alpha}\right) d z \text { for } \lambda \geqslant 0, \\
0 \text { for } \lambda<0,
\end{array}\right.
$$

$\sigma$ being any positive number, and the branch of $z^{\alpha}$ being the principal branch, i.e., $\operatorname{Re}\left(z^{\alpha}\right)>0$ for $\operatorname{Re}(z)>0$.

Let $A$ be a scalar type operator with resolution of the identity $E(\cdot)$, and spectrum contained in the half plane $\{\lambda: \operatorname{Re} \lambda \leqslant 0\}$. Then $A$ generates a strongly continuous uniformly bounded semigroup $\{T(t)\}$, and the calculus described above gives a definition for $(-A)^{\alpha}$.

(11.2) THEOREM. With the same notation as above,

$$
(-A)^{\alpha}=\int_{\sigma(A)}(-\lambda)^{\alpha} d E(\lambda) \text {. }
$$


Proof. $T(t)=\int_{\sigma(A)} e^{t \lambda} d E(\lambda)$, and hence

$$
x^{*} T(t, \alpha) x=\int_{0}^{\infty} \int_{\sigma(A)} f_{t, \alpha}(s) e^{s \lambda} d\left(x^{*} E(\lambda) x\right) d s, \quad x \in X, x^{*} \in X^{*}
$$

But $\left|f_{t, \alpha}(s) e^{s \lambda}\right| \leqslant f_{t, \alpha}(s)$ for all $s \in[0, \infty)$ and $\lambda \in \sigma(A)$, since $f_{t, \alpha}$ is nonnegative. Moreover $\int_{0}^{\infty} f_{t, \alpha}(s) d s=1$ (see [21, p. 262]), hence $f_{t, \alpha}(s) e^{s \lambda}$ is integrable with respect to $d\left|x^{*} E(\lambda) x\right| d s$. So, Fubini's theorem can be used to get

$$
x^{*} \hat{T}(t, \alpha) x=\int_{\sigma(A)} \int_{0}^{\infty} e^{s \lambda} f_{t, \alpha}(s) d s d\left(x^{*} E(\lambda) x\right) .
$$

Direct computation yields $\int_{0}^{\infty} e^{s \lambda} f_{t, \alpha}(s) d s=\exp \left\{-t(-\lambda)^{\alpha}\right\}$. Therefore

$$
\hat{T}(t, \alpha)=\int_{\sigma(A)} \exp \left\{-t(-\lambda)^{\alpha}\right\} d E(\lambda) .
$$

The generator $\hat{A}_{\alpha}$ of the semigroup $\{\hat{T}(t, \alpha): t \geqslant 0\}$ is consequently given by $\hat{A}_{\alpha}=\int_{\sigma(A)}-(-\lambda)^{\alpha} d E(\lambda)$, and hence $(-A)^{\alpha}=-\hat{A}_{\alpha}=\int_{\sigma(A)}(-\lambda)^{\alpha} d E(\lambda)$.

\section{REFERENCES}

1. S. K. Berberian, Notes on spectral theory, Van Nostrand Math. Studies, no. 5, Van Nostrand, Princeton, N. J., 1966. MR 32 \#8170.

2. A. V. Balakrishnan, An operational calculus for infinitesimal generators of semigroups, Trans. Amer. Math. Soc. 91 (1959), 330-353. MR 21 \#5904.

3. E. Berkson, A characterization of scalar type operators on reflexive Banach spaces, Pacific J. Math. 13 (1963), 365-373. MR 27 \#5131.

4. - Semi-groups of scalar type operators and a theorem of Stone, Illinois J.

Math. 10 (1966), 345-352. MR 33 \#583.

5. - Action of $W^{*}$-algebras in Banach spaces, Math. Ann. 189 (1970), 261-

271. MR 43 \#3813.

6. F. F. Bonsall and J. Duncan, Numerical ranges of operators on normed spaces and of elements of normed algebras, London Math. Soc. Lecture Note Ser., no. 2, Cambridge Univ. Press, London and New York, 1971. MR 44 \#5779.

7. A. Devinatz, A note on semi-groups of unbounded self-adjoint operators, Proc.

Amer. Math. Soc. 5 (1954), 101-102. MR 15, 632.

8. N. Dunford and J. T. Schwartz, Linear operators. Parts I, II, III, Interscience, New York, 1958, 1963, 1971. MR 22 \#8302; 32 \#6181.

9. U. Fixman, Problems in spectral operators, Pacific J. Math. 9 (1959), 1029-1051. MR 21 \#7441.

10. S. R. Foguel, The relations between a spectral operator and its scalar part, Pacific J. Math. 8 (1958), 51-65. MR 20 \#3457.

11. C. Foias, On strongly continuous semigroups of spectral operators in Hilbert space, Acta Sci. Math. (Szeged) 19 (1958), 188-191. MR 23 \#A2057; errata, 23, 877.

12. E. Hille and R. S. Phillips, Functional analysis and semi-groups, rev. ed., Amer. Math. Soc. Colloq. Publ., vol. 31, Amer. Math. Soc., Providence, R. I., 1957. MR 19, 664.

13. L. Loomis, An introduction to abstract harmonic analysis, Van Nostrand, New York, 1953. MR 14, 883. 
14. G. Lumer, Semi-inner-product spaces, Trans. Amer. Math. Soc. 100 (1961), 2943. MR 24 \#A2860.

15. - Spectral operators, hermitian operators, and bounded groups, Acta Sci. Math. (Szeged) 25 (1964), 75-85. MR 29 \#6329.

16. C. A. McCarthy, Commuting Boolean algebras of projection. I, Pacific J. Math. 11 (1961), 295-307. MR 23 \#A2750.

17. Commuting Boolean algebras of projections. II. Boundedness in $L_{p}$ Proc. Amer. Math. Soc. 15 (1964), 781-787. MR 30 \#4154.

18. T. W. Palmer, Unbounded normal operators on Banach spaces, Trans. Amer. Math. Soc. 133 (1968), 385-414. MR 37 \#6768.

19. B. Sz.-Nagy, Spektraldarstellung linearer Transformationen des Hilbertschen Raumes, Ergebnisse der Mathematik und ihrer Grenzgebiete, Band 39, Springer-Verlag, Berlin and New York, 1967. MR 35 \#4744.

20. I. Vidav, Eine metrische Kennzeichnung der selbstadjungierten Operatoren, Math. Z. 66 (1956), 121-128. MR 18, 912.

21. K. Yosida, Functional analysis, Die Grundlehren der math. Wissenschaften, Band 123, Springer-Verlag, Berlin, $1965 . \quad$ MR 31 \#5054.

DEPARTMENT OF MATHEMATICS, UNIVERSITY OF ILLINOIS, URBANA, ILLINOIS 61801

DEPARTMENT OF MATHEMATICS, UNIVERSITY OF TORONTO, TORONTO, ONTARIO, CANADA (Current address) 